\title{
Adequate doses of autoantigen administered using the appropriate route may create tolerance and stop autoimmunity
}

\author{
J. Ludvigsson
}

Received: 23 October 2008 / Accepted: 24 October 2008 / Published online: 13 November 2008

(C) Springer-Verlag 2008

Keywords Administration · Autoantigens · Autoimmunity · GAD $\cdot$ Immunotherapy $\cdot$ Tolerance $\cdot$ Type 1 diabetes

To the Editor: Changes in lifestyle and environment may explain the dramatically increased incidence of allergic and autoimmune diseases. Although treatment has become more effective, there are several common autoimmune diseases, such as type 1 diabetes, that pose serious health problems, and we do not know how to cure or prevent these diseases. Different forms of immune intervention may have some, at least transient, effect on the disease process, such as TNF inhibitors in rheumatoid arthritis and anti-CD3 monoclonal antibodies in type 1 diabetes $[1,2]$. However, these interventions often lead to serious adverse events, and there is no method of radically changing the course of the autoimmune process.

Immune therapy involving the subcutaneous injection of the relevant antigen in increasing doses has been shown to be quite effective in stopping the allergic disease process [3], but no effective corresponding immune therapy has been found for autoimmune diseases. Thus, the subcutaneous administration of the autoantigen insulin twice daily to individuals at high risk of developing the disease had no effect [4], and it was not clear whether insulin given orally was beneficial in this respect [5]. Mucosal application of an antigen may have other effects on the immune system, but nasal insulin was found to have no preventive effect against

\footnotetext{
J. Ludvigsson $(\bowtie)$

Division of Paediatrics,

Department of Clinical and Experimental Medicine,

Linköping University,

SE-58185 Linköping, Sweden

e-mail: Johnny.Ludvigsson@lio.se
}

the development of type 1 diabetes in high-risk children [6]. When given subcutaneously, Diapep277, a heat shock protein regarded as an antigen connected to the development of diabetes, seemed to have some slight, so far unconfirmed, effect in adults with type 1 diabetes [7], but had no effect in children [8].

The subcutaneous administration of $20 \mu \mathrm{g}$ of alumformulated GAD65 (GAD65-alum), a major diabetes autoantigen, twice, 1 month apart, to children and adolescents with recent-onset type 1 diabetes has recently produced promising results [9]. At 30 months after the GAD65 vaccination, residual insulin secretion was significantly preserved and there were very clear GAD65-specific effects on the immune system.

This is the first successful result of a new concept in the treatment of autoimmune diseases. It is possible that autoantigen immune therapy may have a similar positive effect on the immune system in other autoimmune diseases. I therefore propose that studies should be planned in which vaccines are formed based on relevant autoantigens. Thus, for type 1 diabetes, not only GAD65, but also alum formulations of insulin, tyrosine phosphatase and perhaps other autoantigens. One can speculate that the autoantigen will be taken up by dendritic cells, which move to draining lymph nodes. There, regulatory $\mathrm{T}$ cells are induced, which in turn migrate to the inflamed part of the body - in the case of type 1 diabetes, the islets of Langerhans - and there inhibit the autoantigen-reactive $\mathrm{T}$ cells.

Thus, studies aimed at preventing the development of type 1 diabetes should investigate the subcutaneous administration of GAD65 together with adjuvant to healthy individuals showing autoimmunity to GAD (i.e. at risk of developing the disease). In line with this, insulin together with adjuvant, perhaps administered at similar doses and at 
similar time intervals as those used for GAD vaccination, should be tried for subcutaneous administration in healthy risk individuals with signs of autoimmunity against insulin. Subcutaneous administration of insulin-alum could also be tried in newly diagnosed diabetic patients. Other relevant autoantigens could be given subcutaneously to individuals with signs of autoimmunity to other autoantigens implicated in the development of multiple sclerosis, rheumatoid arthritis, thyroiditis, etc.

An alternative way of inducing tolerance would be to mimic the successful immune therapy used in the treatment of allergy. The autoantigen should then be given subcutaneously in increasing doses at short intervals until tolerance has developed.

As antigen given subcutaneously has been used with success in allergic diseases, my proposed method for the prevention, and perhaps treatment, of autoimmune diseases may seem simple and may have crossed the mind of many people, but to my knowledge it has not been put forward previously. At a time when we have the tools available both to identify those at risk and to evaluate the effect of any treatment on the immune system, this idea may open new roads for studies aimed at preventing and/or treating not only type 1 diabetes, but several of our severe, so far incurable, diseases.

Duality of interest The author reports receiving grant support from Diamyd Medical for mechanistic studies of GAD-vaccination, and from Novo Nordisk for studies on insulin analogues.

\section{References}

1. Keymeulen B, Vandemeulebroucke E, Ziegler AG et al (2005) Insulin needs after CD3-antibody therapy in new-onset type 1 diabetes. N Engl J Med 352:2598-2608

2. Herold KC, Gitelman SE, Masharani U et al (2005) A single course of anti-CD3 monoclonal antibody hOKT3 $\gamma 1$ (Ala-Ala) results in improvement in C-peptide responses and clinical parameters for at least 2 years after onset of type 1 diabetes. Diabetes 54:1763-1769

3. WHO position paper (1998) Allergy and immunotherapy: therapeutic vaccines for allergic diseases. Geneva: January 27-29 1997. Allergy 53(44 Suppl):1-42

4. Diabetes Prevention Trial-Type 1 Diabetes Study Group (2002) Effects of insulin in relatives of patients with type 1 diabetes mellitus. N Engl J Med 346:1685-1691

5. Skyler JS, Krischer JP, Wolfsdorf J et al (2005) Effects of oral insulin in relatives of patients with type 1 diabetes: The Diabetes Prevention Trial-Type 1. Diabetes Care 28:1068-1076

6. Näntö-Salonen K, Kupila A, Simell S et al (2008) Nasal insulin to prevent type 1 diabetes in children with HLA genotypes and autoantibodies conferring increased risk of disease: a double blind randomised controlled trial. Lancet doi:10.1016/S0140-6736(08)61309-4

7. Raz I, Elias D, Avron A, Tamir M, Metzger M, Cohen IR (2001) Beta cell function in new-onset type 1 diabetes and immunomodulation with a heat shock protein peptide (DiaPep277): a randomised, double-blind, Phase II trial. Lancet 358:1749-1753

8. Schloot NC, Meierhoff G, Lengyel C et al (2007) Effect of heat shock protein peptide DiaPep277 on beta-cell function in paediatric and adult patients with recent-onset diabetes mellitus type 1: two prospective, randomized, double-blind Phase II trials. Diabetes Metab Res Rev 23:276-285

9. Ludvigsson J, Faresjö M, Hjort M et al (2008) GAD treatment and insulin secretion in recent-onset type 1 diabetes. $\mathrm{N}$ Engl $\mathrm{J}$ Med doi:10.1056/NEJMoa0804328 